\title{
DUAL-BEAM ACTUATION OF PIEZOELECTRIC ALN RF MEMS SWITCHES MONOLITHICALLY INTEGRATED WITH ALN CONTOUR-MODE RESONATORS
}

\author{
N. Sinha, R. Mahameed, C. Zuo, M. B. Pisani, C. R. Perez and G. Piazza \\ (The first two authors have contributed equally towards this work) \\ University of Pennsylvania, Philadelphia, Pennsylvania, USA
}

\begin{abstract}
This work reports on piezoelectric Aluminum Nitride (AIN) based dual-beam RF MEMS switches that have been monolithically integrated with AlN contour-mode resonators. The dual-beam switch design presented in this paper intrinsically compensates for the residual stress in the deposited films, requires low actuation voltage $(5-20 \mathrm{~V})$, facilitates active pull-off to open the switch and fast switching times ( 1 to $2 \mu \mathrm{sec})$. This work also presents the combined response (cascaded S-parameters) of a resonator and a switch that were co-fabricated on the same substrate. The response shows that the resonator can be effectively turned on and off by the switch. A post-CMOS compatible process was used for fabrication of both the switches and the resonators. The single-chip RF solution presented herein constitutes an unprecedented step forward towards the realization of compact, low loss and integrated multi-frequency RF front-ends.
\end{abstract}

\section{INTRODUCTION}

The last decade has seen an increase in the efforts for the development of RF MEMS switches that can be implemented in wireless communication systems. RF MEMS switches are known to have many advantages over FET and pin diode switches, such as lower power consumption, improved linearity, higher isolation, and lower insertion loss [1].

Various actuation methods have been employed in RF MEMS switches, such as electrostatic [2-4], piezoelectric [5-8], electromagnetic [9] and thermoelastic [10]. Electrostatic actuation is the most commonly used actuation method. However, this mechanism requires high voltages $(>20 \mathrm{~V}[1])$ that necessitate the presence of a separate on-chip voltage source or charge pump to operate the switch. Reduction in the actuation voltage can be achieved by decreasing the switch stiffness; however, the restoring force that is usually employed for opening the switch and the switching time of the switch both lower with the associated decrease in stiffness. This also affects the ultimate reliability of the switch.

Piezoelectric technology has the ability to meet the requirements of high isolation, low insertion loss, low power consumption and at the same time has the potential to overcome most of the drawbacks encountered in electrostatic switches. Lead Zirconate Titanate (PZT) is the most commonly used piezoelectric material for the development of RF MEMS switches. PZT has very high piezoelectric coefficient values, but the presence of lead makes its fabrication process CMOS incompatible. Few research groups [5-8] have already demonstrated PZT based switches that have the capability of low voltage actuation. AlN films have recently been introduced for RF applications and have demonstrated to be the preferred material for resonator applications [11-14]. AlN offers the ability of direct integration with CMOS over PZT. Furthermore, the monolithic integration of switches and contour-mode resonators [11-12] will tremendously impact the size and power efficiency of any RF front-end by drastically reducing parasitics and off-state leakage. In addition, such integration will enable new RF architectures based on massive arrays of multifrequency AlN contour-mode resonators [14].

In this paper, we present the first AlN based piezoelectric switch that can be operated at low voltages and has been fabricated by building upon the well established process of fabrication of AlN based contour mode resonators and filters (as shown in Fig.1).

\section{DESIGN AND PRINCIPLE OF OPERATION}

The schematic view of the dual-beam AlN piezoelectric switch is shown in Figure 2. The operating principle of each beam is based on the unimorph piezoelectric actuation design (Fig. 3) [15-16]. The routing of the bottom and middle metal layers (the layers that provide the actuation) is done in such a manner that two DC inputs can be used for actuating both beams simultaneously. The stack of layers that make up the unimorph are: one active and one passive layer of AlN, two layers of Platinum and a thick layer of Gold. The (bottom) active layer of AlN is sandwiched between two Platinum electrodes that are used for applying the actuation potential across the piezoelectric layer. The top AlN layer is used to isolate the RF signal from the actuation electrodes. A thick layer of electroplated gold was used on top of the aforementioned AlN stack. The gold layer serves two purposes: firstly as a low resistance carrier for the RF signal, and secondly as a structural layer used for offsetting the neutral axis of the beam needed to produce the bending moment for the switch actuation.

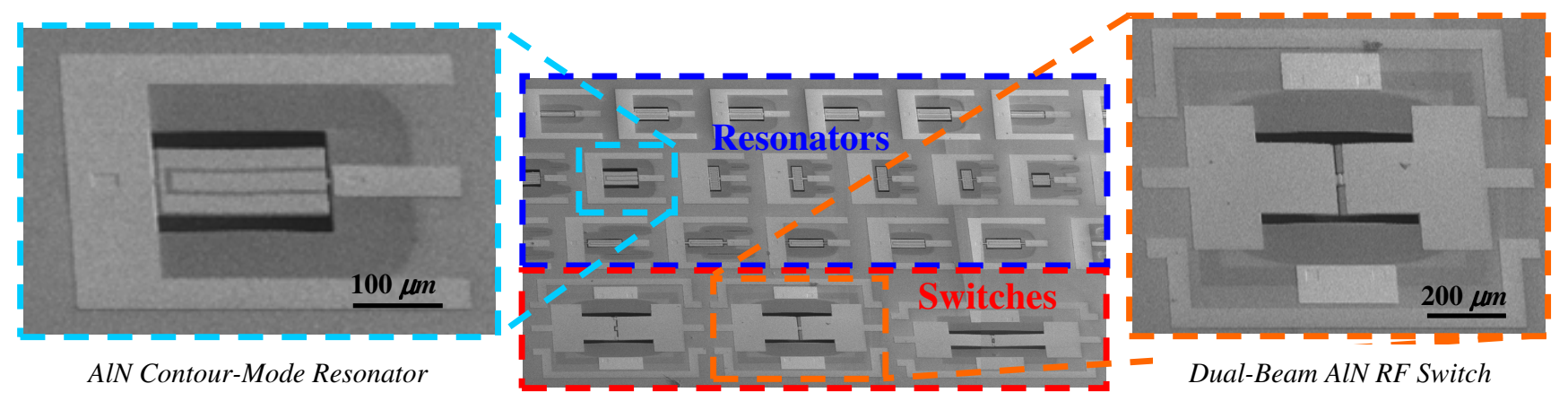

Figure 1: SEM showing dual-beam actuated AlN switches co-fabricated with AlN resonators on the same silicon substrate. The monolithic integration of AlN piezoelectric switches and resonators on a single chip gives us the advantage of achieving new levels of miniaturization for multi-band RF front-ends. 
The switch has a gold-platinum contact and uses an amorphous silicon sacrificial layer for creating the nano (200$300 \mathrm{~nm}$ ) air-gap (Fig. 5).

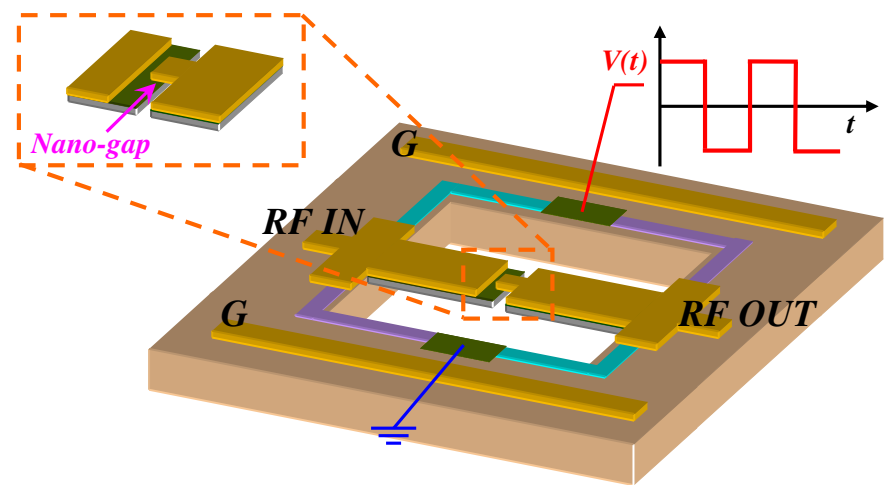

Figure 2: 3-D schematic view of the dual-beam AlN RF switch. This figure illustrates how both arms of the switch can be actuated at the same time. Both mating parts are released, therefore reducing the effect of any residual stress.
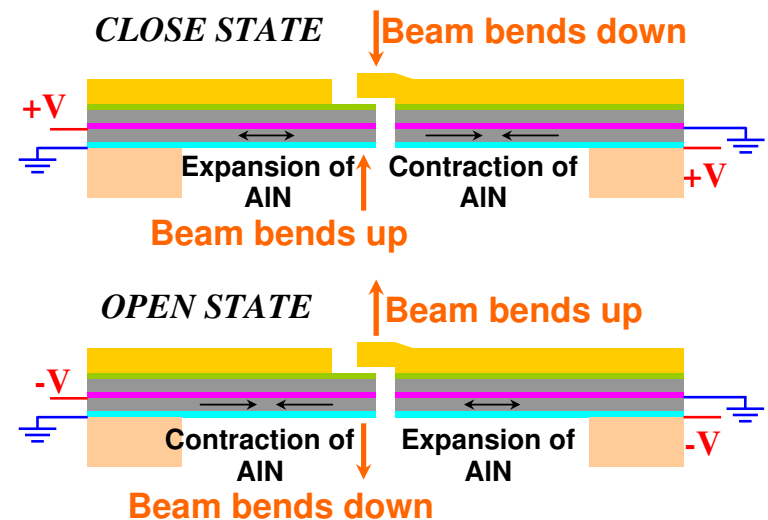

Figure 3: 2-D schematic view of the dual-beam switch showing the operating principle of the switch: The bottom layer of AlN is used for actuation; by reversing the polarity of the applied voltage (done by on-chip electrode routing) the switch can either be opened or closed.

The uniqueness of this work lies in the utilization of the dualbeam mechanism which uses two symmetric released beams for operating the switch. Both beams can be actuated simultaneously, thus giving: (i) low actuation voltage (halved), (ii) high contact forces (doubled for a given voltage), (iii) immunity to residual stress and process-induced variation in the initial nano-gap used to set the RF isolation level, (iv) active pull-off to open the switch and (v) faster switching times (halved).

\section{FABRICATION}

A seven-mask post-CMOS compatible process is used for the co-fabrication of AlN based switches and contour-mode resonators (Fig. 4).

As shown in Figure 4: $1 \mu \mathrm{m}$ of AlN is deposited on top of a $300 \mathrm{~nm}$ insulating layer of low stress nitride (LSN) and $200 \mathrm{~nm}$ of Pt patterned by lift-off. On this first layer of AlN, a second layer of AIN $(1 \mu \mathrm{m})$ is deposited on top of a second layer of Pt $(200 \mathrm{~nm})$.
Opening to the bottom and middle electrodes are made by wetetching of AlN using AZ400k ${ }^{\circledR}$ (a $\mathrm{KOH}$ based developer) at $70^{\circ} \mathrm{C}$. This step shows an improvement in terms of sidewall angle control and reduced surface roughness over [10-11, 13] in defining the via opening. The $\mathrm{AlN}$ is patterned by a $\mathrm{Cl}_{2}$ based dry etch process using $\mathrm{SiO}_{2}$ as hard mask. The top $\mathrm{Pt}(200 \mathrm{~nm})$ layer is deposited and patterned by lift-off to form part of the contact and provide access to the bottom and middle Pt layers. An amorphous silicon layer is evaporated and patterned by lift-off to form the sacrificial layer used to define the nano-gap. A gold seed layer is used for the electroplating of gold and a thick photoresist is employed as a mold for the electroplated layer. After wet etch of the seed layer, the sacrificial layer along with the structure are simultaneously dry-released using $\mathrm{XeF}_{2}$. Dry release is used to eliminate stiction problems that would have otherwise been encountered with wet release techniques. Figure 5 shows a Scanning Electron Micrograph (SEM) of one of the microfabricated switches with a close up view of the nano gap.

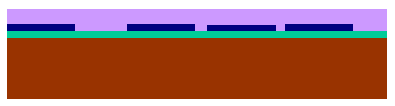

(a)

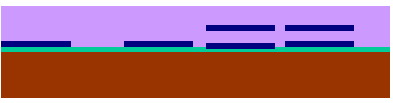

(b)

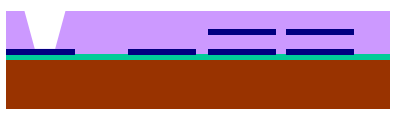

(c)

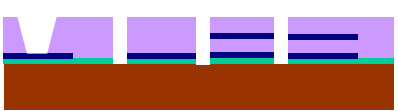

(d)

Si LSN Pt AlN PolySi Gold

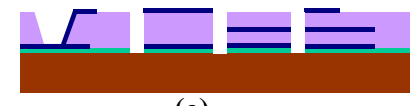

(e)

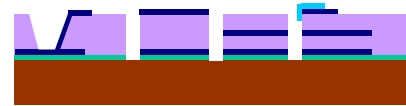

(f)
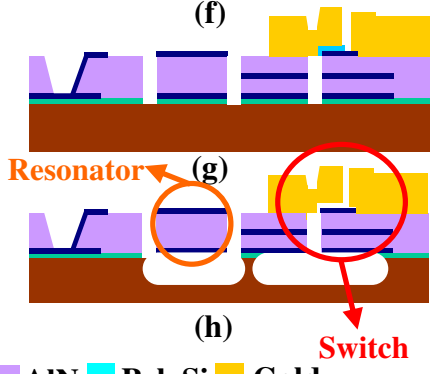

Figure 4: Process flow of the dual-beam switch fabricated with contour-mode resonators. (a) First AlN layer on top of Pt and LSN. (b) Second AlN layer on top of middle Pt. (c) Opening of via to bottom and middle Pt. (d) AlN Etch. (e) Top Pt deposition. (f) Si sacrificial layer. ( $g$ ) Gold Electroplating. (h) $\mathrm{XeF}_{2}$ Release of structures.

\section{EXPERIMENTAL RESULTS}

The experimental characterization of the switch focused on the measurement of the actuator displacement, the on resistance, the switching time and preliminary RF response.

Edge deflection measurements of the actuator were done using a Zygo optical profilometer. Figure 6 shows the edge deflection for a $300 \times 100 \mu \mathrm{m}$ unimorph actuator as function of applied voltage. These measurements (Fig. 6) exhibit very good agreement with FEM simulations $\left(\mathrm{COMSOL}^{\circledR}\right)$.

DC resistance measurements of the switches show that the contact resistance decreases with the applied voltage, proving that higher forces produce lower on-resistance $\left(\mathrm{R}_{\mathrm{on}}\right)$. Our dual-beam actuation scheme takes advantage of this concept and uses two actuators to double the force per unit voltage applied. To further validate this, we tested the change in on-resistance with the applied voltage when either one beam or both beams are actuated (Fig. 7). 

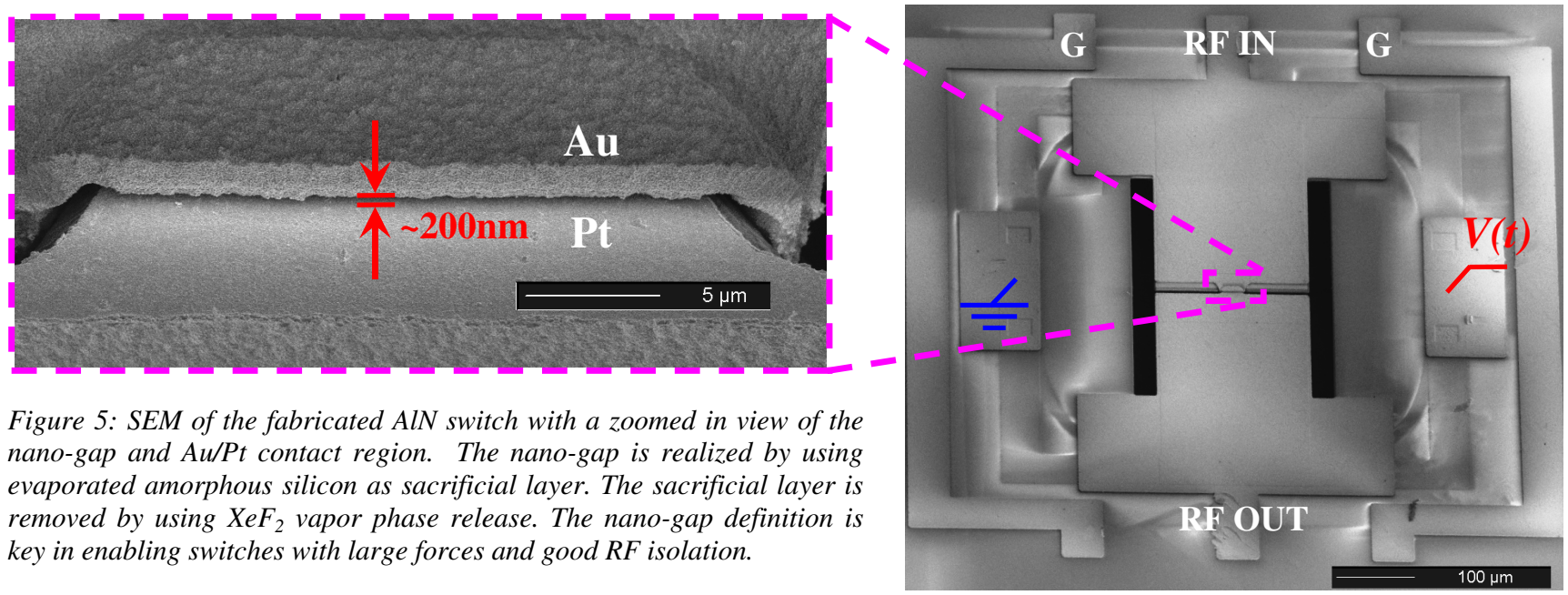

Figure 5: SEM of the fabricated AlN switch with a zoomed in view of the nano-gap and Au/Pt contact region. The nano-gap is realized by using evaporated amorphous silicon as sacrificial layer. The sacrificial layer is removed by using $\mathrm{XeF}_{2}$ vapor phase release. The nano-gap definition is key in enabling switches with large forces and good RF isolation.

The plot in Figure 7 shows that $6.3 \mathrm{~V}$ are needed to establish contact (vs. $12 \mathrm{~V}$ for the single beam) when both beams are actuated and the on-resistance decreases to $\sim 5.4 \Omega$ at $40 \mathrm{~V}$ (vs. $8.4 \Omega$ for a single beam at the same voltage). Some switches exhibited even lower on-resistance $\left(\mathrm{R}_{\mathrm{on}}=3 \Omega\right)$ for the same applied voltage.

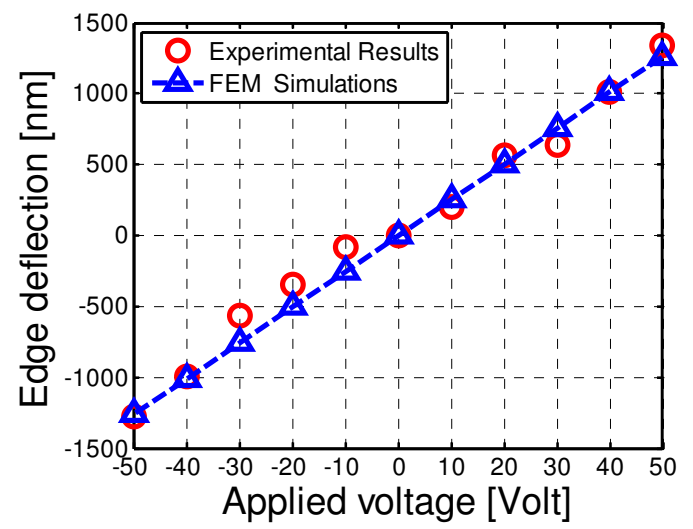

Figure 6: Edge deflection measured by optical profilometer (Zygo) for a $300 \times 100 \mu \mathrm{m}$ unimorph actuator. Experimental results show good agreement with COMSOL FEM simulations.

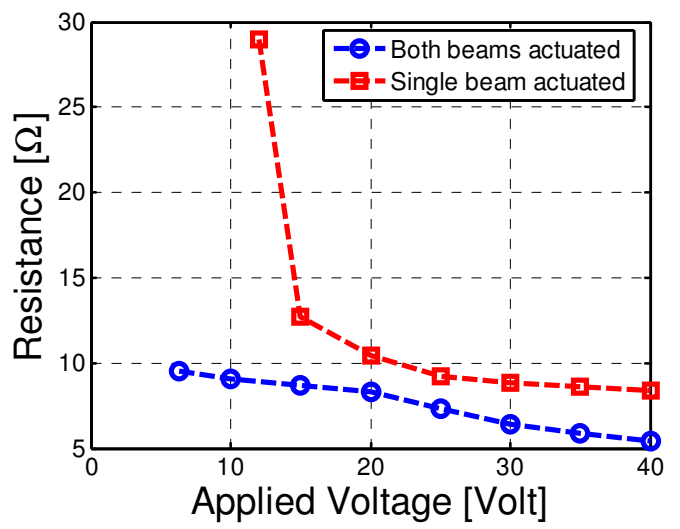

Figure 7: DC on resistance measurements of a switch $(200 \times 200 \mu \mathrm{m})$ for which single and both beams were actuated. The dual beam actuation shows lower resistance for a given voltage.
Preliminary RF measurements were performed in order to verify the RF response of this first AlN switch prototype. Figure 8 presents the isolation and the insertion loss of a 200x200 $\mu \mathrm{m}$ dualbeam AlN switch. In this switch, isolation $>26 \mathrm{~dB}$ and low Return Loss (RL) $<0.75 \mathrm{~dB}$ at $2 \mathrm{GHz}$ were obtained. The current isolation response is limited by a combination of the switch contact and parasitics. Insertion Loss (IL) $<0.67 \mathrm{~dB}$ and $\mathrm{RL}>34 \mathrm{~dB}$ were recorded at $2 \mathrm{GHz}$ for this same switch. The higher than expected IL value is likely due to substrate parasitics, as shown in Figure 8 where the switch response is compared to a through-line on the same substrate. The additional losses are also likely to be due to an unwanted coupling between RF and DC signals and which can be avoided by improved care in the layout of the actuation signal.

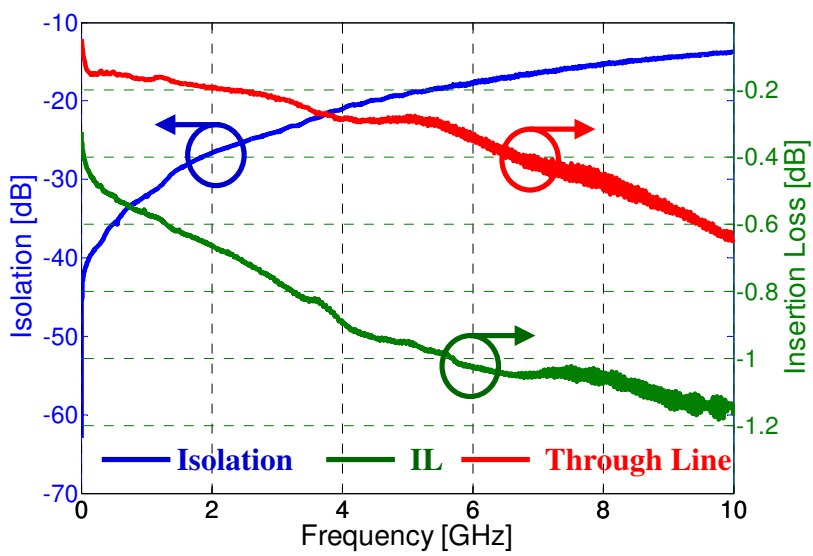

Figure 8: Measurement of isolation and insertion loss (IL) for a $200 \times 200 \mu \mathrm{m}$ dual-beam AlN switch from $100 \mathrm{MHz}$ to $10 \mathrm{GHz}$. Switch IL is compared to the loss in a thru-line (of comparable length).

The same switch was fabricated in conjunction with several AlN contour-mode resonators on the same substrate. Figure 9 shows the combined response (cascaded S-parameters) of a $224 \mathrm{MHz}$ resonator and the switch of Figure 8. The overlap between the resonator response with and without the switch shows that the introduction of the switch does not significantly alter the resonator performance. Furthermore, the switch effectively turns off the resonator by lowering the transmission response by at least $\sim 39.5 \mathrm{~dB}$. 


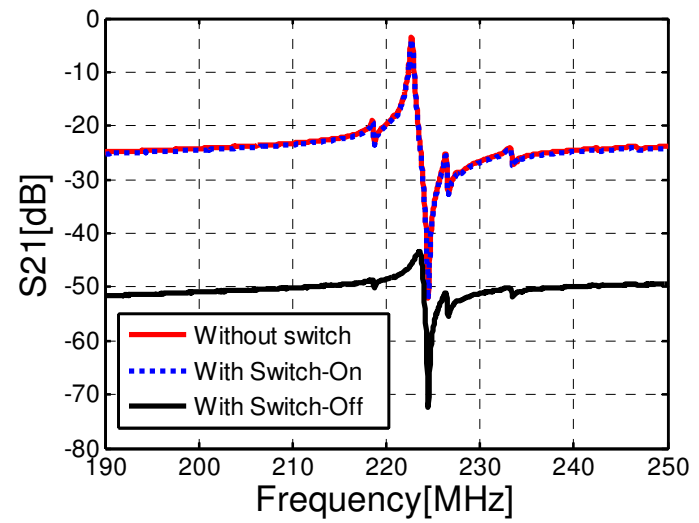

Figure 9: S21 plot (cascaded S-parameters) of a resonator monolithically integrated with the AlN switch presented in this work. The figure shows the response of a resonator with the switch in both on and off states.

In order to experimentally determine the switching time of the AlN switch, a square-wave at $40 \mathrm{~Hz}$ was used to turn the switch on and off, while the change in a DC signal applied across the RF line (i.e. across RF IN and RF OUT of Fig. 5) was monitored to measure the occurrence of contact. The switching time is defined as the time for the output DC signal (i.e. RF OUT, in Fig. 5) to rise from 10 to $90 \%$ of the maximum output signal level in the on state. As shown in Figure 10, a switching time of $\sim 1.6 \mu \mathrm{sec}$ was recorded for the $200 \times 200 \mu \mathrm{m}$ switch. Repeated tests on other switches and at different actuation frequencies and voltages confirmed that the switching time lies between 1 and $2 \mu$ sec.

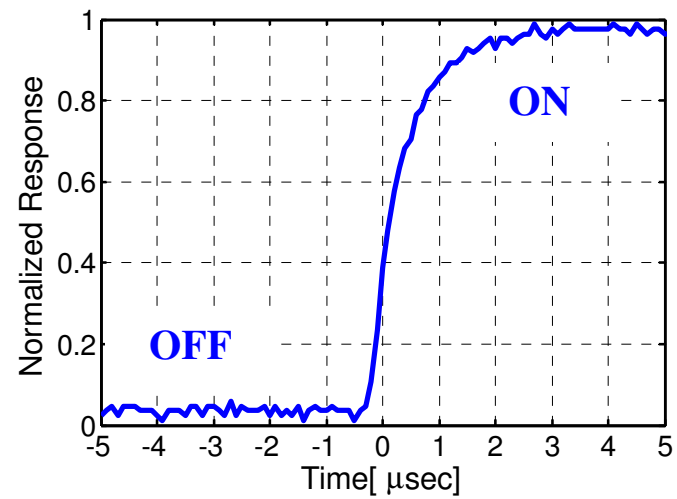

Figure 10: Response of the $200 \times 200 \mu \mathrm{m}$ switch to a square-wave form signal $( \pm 44 \mathrm{~V})$ at $40 \mathrm{~Hz}$.

\section{CONCLUSIONS}

In this work the design, fabrication and experimental testing of the first piezoelectric AlN dual-beam switch monolithically integrated with AIN contour mode resonators has been demonstrated. The RF performance of the first prototype dualbeam switch has been evaluated and shows good isolation $(>26 \mathrm{~dB})$ and $\mathrm{IL}(<0.67 \mathrm{~dB})$ at $2 \mathrm{GHz}$. On-going research is focusing on long-term reliability testing and optimization of the switch in order to reduce IL and actuation voltage. Demonstration of integrated arrays of switches and AIN filters is also in progress.

\section{ACKNOWLEDGEMENTS}

This work was supported by the DARPA ASP Project. The authors will like to acknowledge the help provided by Philip Stephanou and Justin Black at Harmonic Devices Inc. and the staff at the Penn Wolf Nanofabrication Facility.

\section{REFERENCES}

[1] G. M. Rebeiz, "RF MEMS Theory, Design and Technology", $1^{\text {st }}$ Edition, John Wiley \& Sons, Inc., 2003.

[2] P. M. Zavracky, S. Majumder and N. E. McGruer, "Micromechanical switches fabricated using nickel surface micromachining," Journal of MicroElectroMechanical Systems, vol.6, no.1, pp.3-9, Mar 1997.

[3] C. Bozler, R. Drangmeister, S. Duffy, M. Gouker, J. Knecht, L. Kushner, R. Parr, S. Rabe, and L. Travis, "MEMS microswitch arrays for reconfigurable distributed microwave components", IEEE MTT-S International Microwave Symposium Digest, Boston, MA (June 2000), pp. 153-156.

[4] S. Duffy, C. Bozler, S. Rabe, J. Knecht, L. Travis, P. Wyatt, C. Keast, and M. Gouker, "MEMS microswitches for reconfigurable microwave circuitry", Microwave and Wireless Comp. Lett., 11/3/2001, pp. 106-108.

[5] H. C. Lee, J. H. Park, J. Y. Park, H. J. Nam and J. U. Bu "Design, fabrication and RF performances of two different types of piezoelectrically actuated Ohmic MEMS switches", Journal of Micromechanics Microengineering, 15(2004), pp. 2098-2104.

[6] J. H. Park, H. C. Lee, Y. H. Park, Y. D. Kim, C. H. Ji, J. Bu and H. J. Nam, "A fully wafer-level packaged RF MEMS switch with low actuation voltage using a piezoelectric actuator", Journal of Micromechanics Microengineering 16(2006), pp. 2281-2286.

[7] S. J. Gross, S. Tadigadapa, and T. N. Jackson, S. Trolier-McKinstry, Q. Q. Zhang "Lead-Zirconate-Titanate-Based Piezoelectric Micromachined Switch", Appl. Phys. Lett., 83/1/2003, pp. 174-176.

[8] R. G. Polcawich, D. Judy, J. S. Pulskamp, S. Trolier-McKinstryand M. Dubey," "Advances in Piezoelectrically Actuated RF MEMS Switches and Phase Shifters", Microwave Symposium, (2007) IEEE/MTT-S International, pp.2083-2086.

[9] M. Ruan, J. Shen, and C. B. Wheeler, "Latching Micromagnetic Relays", Journal of MicroElectroMechanical. Systems, 10/2001, pp. 511-517.

[10] R. D. Streeter, C. A. Hall, R. Wood, and R. Madadevan, "VHF highpower tunable RF bandpass filter using microelectromechanical (MEM) microrelays", Int. J. RF Microwave CAE, 11/5/2001, pp. 261-275.

[11] G. Piazza, P. J. Stephanou and A. P. Pisano, "Piezoelectric Aluminum Nitride Vibrating Contour-Mode MEMS Resonators", Journal of MicroElectroMechanical Systems, 15/6/2006, pp. 1406-1418.

[12] G. Piazza, P. J. Stephanou and A. P. Pisano, "Single-Chip MultipleFrequency AlN MEMS Filters Based on Contour-Mode Piezoelectric Resonators", Journal of MicroElectroMechanical Systems, 16/2/2007, pp. 319-328.

[13] R. Ruby, P. Bradley, J. Larson III, Y. Oshmyansky and D. Figueredo, "Ultra-miniature high-Q filters and duplexers using FBAR technology ", Solid-State Circuits Conference, 2001. Digest of Technical Papers. ISSCC. 2001 IEEE International, pp.120-121, 438.

[14] G. Piazza, P. J. Stephanou and A. P. Pisano, "One and two port Piezoelectric Higher Order Contour-mode MEMS Resonators for Mechanical Signal Processing", Solid State Electronics, 51/ 1112/2007, pp. 1596-1608.

[15] J. G. Smits, S. Dalke and T. K. Cooney, "The Constituent Equations of Piezoelectric Bimorphs", Sensors and Actuators A, 28/1991, pp. 41-61.

[16] D. L. DeVoe and A. P. Pisano, "Modeling and optimal design of piezoelectric cantilever microactuators", Journal of MicroElectroMechanical Systems, 6/3/1997, pp. 266-70. 